Research Article

\title{
Evaluation and Comparison of Medical Students Stressors and Coping Strategies among Undergraduate Preclinical and Clinical Year Students Enrolled in Medical School of Arsi University, Southeast Ethiopia
}

\author{
Leta Melaku $(1)$ and Guta Bulcha \\ Department of Biomedical Sciences, College of Health Sciences, Arsi University, Asella, Oromia, Ethiopia \\ Correspondence should be addressed to Leta Melaku; letamelaku@gmail.com
}

Received 19 July 2021; Revised 30 October 2021; Accepted 9 November 2021; Published 22 November 2021

Academic Editor: Enrique Palou

Copyright (C) 2021 Leta Melaku and Guta Bulcha. This is an open access article distributed under the Creative Commons Attribution License, which permits unrestricted use, distribution, and reproduction in any medium, provided the original work is properly cited.

\begin{abstract}
Background. The transition into higher education is stressful as university students face many stressful events. Medical students must deal with stressors specific to medical education. While many students adjust effectively to the university context, large proportions of students are at risk of developing mental health problems. The objective of the present study was to evaluate the most common medical student's stressors and coping strategies among undergraduate students enrolled in the Medical School of Arsi University and their association with educational year levels. Methods. An Institutional based cross-sectional study was conducted on 265 medical students by systematic random sampling. Data were collected by pretested self-administrative questionnaire and analyzed by SPSS-21 software. Logistic regression analysis was employed, and statistical significance was accepted at $p<0.05$. Result. In the present study, 5 questionnaires were rejected for incompleteness, giving a response rate of $98.1 \%$. The top sources of stress were lack of time to review, conflict with teacher(s), and uncertainty of what was expected. ARS domain was the main cause of high stress, followed by IRS and TLRS. Religious coping, active coping, positive reframing, and planning were the most commonly used coping strategies. When preclinical year students were compared with the clinical years, TLRS and DRS domains were identified as the most common cause of stress in the preclinical years. Furthermore, instrumental support, behavioral disengagement, acceptance, religion, self-blame, and emotional support were the most commonly used coping strategies in the preclinical years compared to the clinical years. Conclusion. We observed that academic-related stressors followed by interpersonal and intrapersonal stressors are the major stressors faced by students. Active coping strategies were the most commonly employed ones rather than avoidant strategies. Stress reduction interventions were recommended.
\end{abstract}

\section{Introduction}

The transition from childhood to young adulthood is often marked by the beginning of studying in university [1], and the transition into higher education is a stressful time as university students face many stressful events [2]. Personal and environmental events that cause stress are known as stressors [3]. Stress is a feeling that is initiated when a person perceives that demands exceed the resources mobilized by the individual [4]. When stress enhances physical or mental function, it may be considered eustress [5]. Most people are more active, invigorated, creative, and productive because of eustress [6]. Conversely, persistent stress that is not resolved through coping or adaptation, deemed distress, has been shown to cause physical and mental health problems and reduced self-esteem and may affect academic achievement and personal and professional development $[7,8]$. Some variance in stress responses and consequences may be attributable to characteristics of the stressor, and some events are nearly universal in evoking a state of stress [9]. While some stress may enhance academic performance [10], high levels of stress among medical students are associated with 
depression [11-13], burnout [14, 15], and somatic complaints [13]. Medical students report higher levels of psychological distress than their same-age peers [16-21], despite having similar or healthier profiles than peers at the outset of medical school [11,22-24]. Studies have indeed documented that stress levels increase during medical school, peaking either in the second year [11] or when students enter the medical wards [25]. Previous research has explored students' vulnerability to stress $[19,26,27]$. Several studies have documented major stressors for medical students, including academics [15, 28], lack of balance [29], relationships [28], poor student guidance/support [29], volume of information $[28,29]$, finances [29], uncertainty of the future [15], lack of time to oneself [28], time and responsibility [29], the need to succeed [28], and frequent academic examinations in a competitive environment [7]. Furthermore, students living in the dormitory might be susceptible to additional stressors like financial issues [30], adaptation to the new environment, being away from home for the first time, and changes in living arrangements [31]. The educational system also plays an enabling role, subsequently leading to increased stress levels experienced by students. Some of the sources include overcrowded lecture halls, semester grading system, inadequate resources and facilities [32], vastness of the syllabus $[32,33]$, long hours, and expectations of rote learning [34]. Parents and institutions relentlessly instill the fear of failure, which affects their self-esteem and confidence [35]. Healthcare systems usually also provide many stimuli that produce stress due to contact with illnesses, pain, suffering, disability, patients, and death, as well as the fact of developing a new role they are not completely prepared for [36]. In many medical schools, the environment itself is a prevailing pressure situation, providing an authoritarian and rigid system. In general, a recent review described six major themes associated with student distress: adjustment, ethical concerns, exposure to patient death and suffering, student mistreatment, personal life events, and educational debt [37].

The overall impact of a stressor will depend on its characteristics and the characteristics of those who have been affected. The same stressors may be perceived differently by different medical students, depending on their cultural background, personal traits, experience, and coping skills [3]. Therefore, stress can be thought of as a state resulting from an "imbalance between demands and resources" or as occurring when "pressure exceeds one's perceived ability to cope" [38]. Coping with stress is important for human survival [39] and has been viewed as a stabilizing factor that may assist an individual in psychosocial adaptation during stressful events [40]. In addition to coping with stressors of everyday life, medical students must deal with stressors specific to medical education. Various coping strategies have been studied for medical students to reduce the level of stress, including effective time management, social support, positive reappraisal, engagement in leisurely pursuits and mindfulness-based stress reduction classes, wellness electives, informal support groups, and mentoring programs [33, 41, 42]. While many university students adjust effectively to the university context, a large proportion of students are adversely impacted by stress and are at risk of developing mental health problems [43]. Failure in coping with stress may lead students to stop or discontinue further education, have suicidal intentions, or participate in activities such as smoking, drinking, aggressive behavior with others, or damaging the institution's property, or be involved in violent activities by disrespecting laws and rights of others $[21,22]$. There is also emotion-based coping that involves accepting responsibility and self-blame. This type of coping is useful in the first year of medical school. In contrast, in later years, the trend shifted towards confronting, cognitive, and planned problem-solving [44-47].

Arsi University College of Health Sciences focuses on educating/training competent and ethical health professionals for the contribution of paramount in national GDP, particularly the health of the whole community in the growing manufacturing industry, at all levels [48]. In the 2019 GC academic calendar, the regular undergraduate programs of the College of Health Sciences includes 576 medical, 125 public health, 154 clinical nursing, 40 operating theater nurse, 27 neonatal nursing, 75 anesthesia, 152 pharmacies, 108 medical laboratory sciences, and 120 midwifery students at different levels of study with a total of 1377 active students. The medical curriculum of the School of Medicine at Arsi University takes six years [48]. Medical students stay three years in the preclinical and three in the clinical practice. Therefore, the main aim of the present study was to evaluate the most common medical students' stressors and coping strategies among undergraduate students enrolled in the Medical School of Arsi University and their association with educational year levels.

\section{Methods and Materials}

Institutional based cross-sectional study was conducted starting from January 10 to 30, 2019, among 265 sampled undergraduate medical students of Arsi University. Since the incidence of stress and stress-related illnesses such as anxiety and depression among medical students is increasingly reported in the literature [49], the sample size of the present study was first estimated using a single population proportion with an assumption of $95 \% \mathrm{CI}$, 5\% margin of error, and prevalence of depression (51.3\%), anxiety (66.9\%), and stress (53\%), which is taken from [50]. The overall numbers of Arsi University medical students were $<10,000$. Therefore, by taking the maximum result of sample size obtained from a single population proportion, the minimum required sample size for the present study was calculated through the correction formula. After proportional allocation of the calculated sample size to each academic year level, the stratified random sampling method was employed. From each stratum, by randomly selecting the first students based on their ID number, respondents were chosen every 4 intervals through a systematic random sampling method. All medical students undergoing training in the Arsi University at the time of the study were eligible to participate with the exceptions of severely ill students and being out of town during the time of data collection. Of the 265 sampled students learning at Arsi University, 260 (98.1\%) agreed to take part. 
An ethical support letter was obtained from the Arsi University ethical board. Informed consent was taken from all respondents during data collection, and participation was totally voluntary. Confidentiality was kept unanimously. Data were collected by using manually distributed self-administered questionnaires that comprised of the following parts:

(i) Sociodemographic profiles: Age, gender, marital status, residence, academic year, income, religion, and ethnicity. In the present study, to undertake the comparison, academic years 1-3 were classified as a preclinical educational year level and coded as " 0 " whereas those from academic years 4-6 were classified as a clinical educational year level and coded as "1."

(ii) Medical Student Stressor Questionnaire-20 (MSSQ-20): 20 items grouped into six stressor domains for identifying the source and measuring the severity of stress experienced by medical students in the past six months [51]. These stressor domains are academic-related stressors (ARS), interpersonal and intrapersonal-related stressors (IRS), related social stressors (SRS), teaching and learning-related stressors (TLRS), desire and drive-related stressors (DRS), and group activity-related stressors (GARS). Although the status of each stressor determines the degree of stress experienced, factors that showed higher mean values from all domains were listed as the top sources of stress. Respondents were asked to respond by choosing from: "causing no stress at all," "causing mild stress," "causing moderate stress," "causing high stress," and "causing severe stress." The MSSQ is scored by assigning a value of zero to four for each of the respective responses. A number of studies reported that the MSSQ was found to have strong internal consistency [3, 51-53].

(iii) Brief COPE scale: It is designed to assess some different coping behaviors and thoughts after a person's response to a specific situation among adults with or without clinical conditions $[54,55]$. It consists of 28 items, and each item is rated on a 4 point Likert scale ranging from "I have not been doing this at all (score 1)" to "I have been doing this lot (score 4)." The items were scored to produce 14 dimensions (minimum mean score was 2, and maximum score was 8 ), and each dimension reflects the use of a coping strategy such as active coping, planning, acceptance, denial, self-distraction, use of substance, use of emotional support, use of instrumental support, behavioral disengagement, venting, positive reframing, humor, religion, and self-blame [54]. Mean score interpretations were as follows: $2.00=$ has not been doing this at all, 2.01 to $4.00=$ has been doing this a little, 4.01 to $6.00=$ has been doing this medium amount, and 6.01 to $8.00=$ has been doing this lot. The higher score indicates greater coping by the respondents [56]. It is a validated instrument in which Cronbach's alpha values range $0.50-0.90$, with only 3 coping strategies falling below $0.60[54,56]$.

The questionnaire was pretested on 13 randomly selected undergraduate medical students of Hawasa University for making the questionnaires' content, wording, and instructions, and ease of completion more understandable for respondents. After checking the collected data for completeness, it was double entered into EpiData version 3.1 (McGraw-Hill/Irwin (C2009) and exported into SPSS version 21 (IBM Corp., Armonk, NY) for analysis. Incomplete and inconsistent data were excluded from the analysis. The data were processed using descriptive analysis, including frequency distribution, cross tabulation, and summary measures. Bivariate logistic regression was used to measure the association between independent variables with dependent variables. Statistical significance was accepted at $p<0.05$.

\section{Results}

3.1. Sociodemographic Characteristics. Out of 265 sampled undergraduate medical students of Arsi University, 5 questionnaires were rejected for incompleteness, giving a response rate of $98.1 \%$. Therefore, the present study involved 260 respondents who had a range of age between 18 and 27 with a mean SD of $22.03(+2.62)$ years. In the present study, most respondents were male $(63.1 \%)$, single in marital status (75.4\%), and living in campus (91.5\%), with a monthly income of $<700$ ETB (52.7\%). Regarding their academic year, religion, and ethnicity, 54 (20.8\%) were from the sixth year, 130 (50\%) were Orthodox believers, and 139 (53.9\%) were Oromo, respectively (Table 1).

3.2. Sources and Severity of Stressors of Medical Students. According to the MSSQ-20 score, the overall top sources of stress from the 20 items of MSSQ were lack of time to review what has been learned, conflict with teacher(s), uncertainty of what is expected, facing illness or death of the patients, unwillingness to study medicine, and need to do well (Table 2).

In the present study, 20 items of MSSQ were also grouped into six main stressor domains of medical students and concerning the severity of stress experienced by each stressor domain. DRS was identified as the main cause of the mild level of stress $(121(46.5 \%))$, which is followed by GARS (106 $(40.8 \%))$, TLRS (91 (35.0\%)), SRS (81 (31.2\%)), IRS (67 $(25.8 \%))$, and ARS $(59(22.7 \%))$. In addition, SRS was identified as the main cause of the moderate level of stress (102 (39.2\%)), which is followed by ARS (97 (37.3\%)), GARS (95 (36.5\%)), IRS (87 (33.5\%)), and lastly both DRS and TLRS (69 $(26.5 \%))$. On the other hand, the majority of the respondents (82 (31.5\%)) considered ARS to be the cause of high stress which was followed by IRS (79 $(30.4 \%))$, TLRS (73 $(28.1 \%))$, SRS (55 (21.2\%)), GARS (44 (16.9\%)), and DRS (36 (13.8\%)). Furthermore, the majority of the respondents (34 (13.1\%)) considered DRS to be the cause of severe stress which was followed by both IRS and TLRS (27 (10.4\%)), then both ARS and SRS (22 (8.5\%)), and lastly GARS (15 (5.8\%)) (Figure 1). 
TABLE 1: Sociodemographic characteristics of study participants in relation to gender, AU, January 2019.

\begin{tabular}{|c|c|c|c|c|}
\hline \multicolumn{2}{|c|}{ Sociodemographic variables } & Male & Female & Total $(N=260)$ \\
\hline \multirow{3}{*}{ Age } & $<20$ years & $36(54.5 \%)$ & $30(45.5 \%)$ & $66(100.0 \%)$ \\
\hline & $20-24$ years & $91(67.4 \%)$ & $44(32.6 \%)$ & $135(100.0 \%)$ \\
\hline & $>24$ years & $37(62.7 \%)$ & $22(37.3 \%)$ & $59(100.0 \%)$ \\
\hline \multirow{2}{*}{ Marital status } & Single & $128(65.3 \%)$ & $68(34.7 \%)$ & $196(100.0 \%)$ \\
\hline & Married & $36(56.3 \%)$ & $28(43.8 \%)$ & $64(100.0 \%)$ \\
\hline \multirow{2}{*}{ Monthly income } & $\leq 700 \mathrm{ETB}$ & $87(63.5 \%)$ & $50(36.5 \%)$ & $137(100.0 \%)$ \\
\hline & $>700$ ETB & $77(62.6 \%)$ & $46(37.4 \%)$ & $123(100.0 \%)$ \\
\hline \multirow{6}{*}{ Educational year } & $1^{\text {st }}$ year & $27(61.4 \%)$ & $17(38.6 \%)$ & $44(100.0 \%)$ \\
\hline & $2^{\text {nd }}$ year & $22(48.9 \%)$ & $23(51.1 \%)$ & $45(100.0 \%)$ \\
\hline & $3^{\text {rd }}$ year & $23(59.0 \%)$ & $16(41.0 \%)$ & $39(100.0 \%)$ \\
\hline & $4^{\text {th }}$ year & $36(76.6 \%)$ & $11(23.4 \%)$ & $47(100.0 \%)$ \\
\hline & $5^{\text {th }}$ year & $23(74.2 \%)$ & $8(25.8 \%)$ & $31(100.0 \%)$ \\
\hline & $6^{\text {th }}$ year & $33(61.1 \%)$ & $21(38.9 \%)$ & $54(100.0 \%)$ \\
\hline \multirow{2}{*}{ Residency } & Nondormitory & $11(50.0 \%)$ & $11(50.0 \%)$ & $22(100.0 \%)$ \\
\hline & Dormitory & $153(64.3 \%)$ & $85(35.7 \%)$ & $238(100.0 \%)$ \\
\hline \multirow{4}{*}{ Religion } & Orthodox & $80(61.5 \%)$ & $50(38.5 \%)$ & $130(100.0 \%)$ \\
\hline & Muslim & $40(62.5 \%)$ & $24(37.5 \%)$ & $64(100.0 \%)$ \\
\hline & Protestant & $42(67.7 \%)$ & $20(32.3 \%)$ & $62(100.0 \%)$ \\
\hline & Others ${ }^{*}$ & $2(50.0 \%)$ & $2(50.0 \%)$ & $4(100.0 \%)$ \\
\hline \multirow{7}{*}{ Ethnicity } & Oromo & $97(69.8 \%)$ & $42(30.2 \%)$ & $139(100.0 \%)$ \\
\hline & Amhara & $38(53.5 \%)$ & $33(46.5 \%)$ & $71(100.0 \%)$ \\
\hline & Sidama & $2(33.3 \%)$ & $4(66.7 \%)$ & $6(100.0 \%)$ \\
\hline & Tigre & $7(50.0 \%)$ & $7(50.0 \%)$ & $14(100.0 \%)$ \\
\hline & Wolayita & $5(62.5 \%)$ & $3(37.5 \%)$ & $8(100.0 \%)$ \\
\hline & Gurage & $12(66.7 \%)$ & $6(33.3 \%)$ & $18(100.0 \%)$ \\
\hline & Others $^{\dagger \dagger}$ & $3(75.0 \%)$ & $1(25.0 \%)$ & $4(100.0 \%)$ \\
\hline
\end{tabular}

${ }^{¥}$ Catholic, Waqefatta, Faith, and Pagan. ${ }^{\dagger \dagger}$ Silte, Harari, and Kambata. ETB = Ethiopian Birr. Current exchange rate: $\$ 1$ USD $=28.05$ ETB.

3.3. Effectiveness of Coping Strategies. In the present study, the total coping strategies applied by respondents were 28 , with a minimum score of 2 and a maximum score of 8 . Among coping strategies, "religious coping with a mean $( \pm$ SD) coping score of 5.94 (1.85), active coping with 5.52 (1.75), positive reframing with 5.39 (1.67), and planning with 5.38 (1.71)" were found to be the axioms used by most respondents. Inversely, "behavioral disengagement with a mean $( \pm S D)$ coping score of 4.34 (1.75), denial with 4.10 (1.70), and substance use with 3.47 (1.81)" were found to be the least used coping strategies (Table 3 ). In the present study, active coping strategies were the most commonly used strategies compared to avoidant strategies. However, there was a variation across genders concerning the specific types of coping strategies used by respondents. Females were more commonly involved in the use of emotional support with a mean $( \pm S D)$ coping score of 4.89 (1.67), use of instrumental support with 5.33 (1.82), positive reframing with 5.65 (1.77), humor with 4.93 (1.89), acceptance with 5.15 (1.78), religion with 6.09 (2.00), and self-blame with 4.78 (1.64) than males. Contrariwise, males more commonly used active coping with a mean $( \pm S D)$ coping score of 5.56 (1.71) and substance use with 3.49 (1.75) than females (Table 3).

3.4. Association of Medical student stressors with Educational Year Level. A binary logistic regression analysis was applied to evaluate the possible impact of the study participant's stressor domain on the educational year. From the total six domains of medical student stressors, 4 stressor domains were not statistically associated with respondents' educational year (Table 4). However, TLRS and DRS domains had a significant statistical association with years of medical education $(p<0.05)$. In the present study, TLRS were a 1.90-time more common cause of stress in the preclinical years than in clinical years $(95 \% \mathrm{Cl}$ : 1.14-3.15). Similarly, DRS were a 1.55 -time more common cause of stress in the preclinical years than in clinical years (95\% Cl: 1.09-2.69).

3.5. Association of Medical Student Coping Strategies with Educational Year Level. A binary logistic regression analysis was applied to evaluate the possible impact of the study participant's coping strategies on the year of education. From the total 14 factors of the BC instrument, 8 factors were not statistically associated with respondents coping strategies (Table 5). However, the use of instrumental support, behavioral disengagement, acceptance, religion, self-blame, and emotional support had a significant statistical association with years of medical education $(p<0.05)$. For instance, the use of instrumental support was 3.01-time more common coping strategy in the preclinical years compared to clinical years (95\% Cl: 1.15-7.89). Behavioral disengagements were a 2.18-time more common cause of stress in the preclinical years compared to clinical years $(95 \%$ $\mathrm{Cl}$ : 1.14-4.15). Acceptance was also another coping strategy 
TABLE 2: Sources of stress among study participants from items of medical student stressors, $N=260$, AU, January 2019.

\begin{tabular}{|c|c|c|c|c|c|c|c|}
\hline \multirow[b]{2}{*}{$\begin{array}{l}\text { Stressor } \\
\text { domain }\end{array}$} & \multirow[b]{2}{*}{ Items } & \multicolumn{5}{|c|}{ MSSQ score N (\%) } & \multirow[b]{2}{*}{ Mean (SD) } \\
\hline & & $\begin{array}{l}\text { No stress at } \\
\text { all }\end{array}$ & $\begin{array}{l}\text { Mild } \\
\text { stress }\end{array}$ & $\begin{array}{c}\text { Moderate } \\
\text { stress }\end{array}$ & $\begin{array}{l}\text { High } \\
\text { stress }\end{array}$ & $\begin{array}{c}\text { Severe } \\
\text { stress }\end{array}$ & \\
\hline \multirow{5}{*}{ ARS } & Tests/examinations & $33(12.7)$ & $76(29.2)$ & $85(32.7)$ & $39(15.0)$ & $27(10.4)$ & $1.81(1.16)$ \\
\hline & Falling behind in reading schedule & $25(9.6)$ & $93(35.8)$ & $75(28.8)$ & $51(19.6)$ & $16(6.2)$ & $1.77(1.07)$ \\
\hline & $\begin{array}{c}\text { The large amount of content to be } \\
\text { learned }\end{array}$ & $31(11.9)$ & $73(28.1)$ & $72(27.7)$ & $60(23.1)$ & $24(9.2)$ & $1.90(1.16)$ \\
\hline & $\begin{array}{c}\text { Lack of time to review what has been } \\
\text { learned }\end{array}$ & $24(9.2)$ & $64(24.6)$ & 77 (29.6) & $67(25.8)$ & $28(10.8)$ & $2.04(1.14)$ \\
\hline & Heavy workload & $32(12.3)$ & $70(26.9)$ & $78(30.0)$ & $62(23.8)$ & $18(6.9)$ & $1.86(1.12)$ \\
\hline \multirow{4}{*}{ IRS } & $\begin{array}{l}\text { Verbal/physical/abuse by other } \\
\text { student(s) }\end{array}$ & $61(23.5)$ & $92(35.4)$ & $53(20.4)$ & $42(16.2)$ & $12(4.6)$ & $1.43(1.15)$ \\
\hline & Verbal/physical/abuse by teacher(s) & $46(17.7)$ & $57(21.9)$ & $55(21.2)$ & $65(25.0)$ & $37(14.2)$ & $1.96(1.32)$ \\
\hline & Verbal/physical/abuse by personnel(s) & $55(21.2)$ & $64(24.6)$ & $64(24.6)$ & $59(22.7)$ & $18(6.9)$ & $1.70(1.23)$ \\
\hline & Conflict with teacher(s) & $43(16.5)$ & $29(11.2)$ & $61(23.5)$ & $75(28.8)$ & $52(20.0)$ & $2.25(1.35)$ \\
\hline \multirow{3}{*}{ TLRS } & Not enough feedback from teacher(s) & $57(21.9)$ & $68(26.2)$ & $76(29.2)$ & $39(15.0)$ & $20(7.7)$ & $1.60(1.20)$ \\
\hline & Uncertainty of what is expected of me & $35(13.5)$ & $77(29.6)$ & $78(30.0)$ & $50(19.2)$ & $20(7.7)$ & $1.78(1.14)$ \\
\hline & Lack of recognition for work done & $53(20.4)$ & $68(26.2)$ & $70(26.9)$ & $47(18.1)$ & $22(8.5)$ & $1.68(1.23)$ \\
\hline \multirow{3}{*}{ SRS } & $\begin{array}{l}\text { Unable to answer questions from } \\
\text { patients' }\end{array}$ & $49(18.8)$ & $57(21.9)$ & $65(25.0)$ & $59(22.7)$ & $30(11.5)$ & $1.86(1.28)$ \\
\hline & $\begin{array}{c}\text { Talking to patients about personal } \\
\text { problems }\end{array}$ & $73(28.1)$ & $68(26.2)$ & $54(20.8)$ & $41(15.8)$ & $24(9.2)$ & $1.52(1.30)$ \\
\hline & Facing illness or death of the patients & $46(17.7)$ & $55(21.2)$ & $56(21.5)$ & $62(23.8)$ & $41(15.8)$ & $1.99(1.34)$ \\
\hline \multirow{2}{*}{ DRS } & Unwillingness to study medicine & $67(25.8)$ & $64(24.6)$ & $50(19.2)$ & $39(15.0)$ & $40(15.4)$ & $1.70(1.40)$ \\
\hline & Parental wish for you to study medicine & $86(33.1)$ & $64(24.6)$ & $52(20.0)$ & $27(10.4)$ & $31(11.9)$ & $1.43(1.36)$ \\
\hline \multirow{3}{*}{ GARS } & Participation in class presentation & $68(26.2)$ & $69(26.5)$ & $74(28.5)$ & $33(12.7)$ & $16(6.2)$ & $1.46(1.18)$ \\
\hline & Need to do well (imposed by others) & $46(17.7)$ & $92(35.4)$ & $73(28.1)$ & $37(14.2)$ & $12(4.6)$ & $1.53(1.08)$ \\
\hline & The feeling of incompetence & $69(26.5)$ & $74(28.5)$ & $51(19.6)$ & $47(18.1)$ & $19(7.3)$ & $1.51(1.26)$ \\
\hline
\end{tabular}

ARS: academic-related stressors; IRS: interpersonal- and intrapersonal-related stressors; TLRS: teaching- and learning-related stressors; SRS: social-related stressors; DRS: drive- and desire-related stressors; GARS: group activity-related stressors.

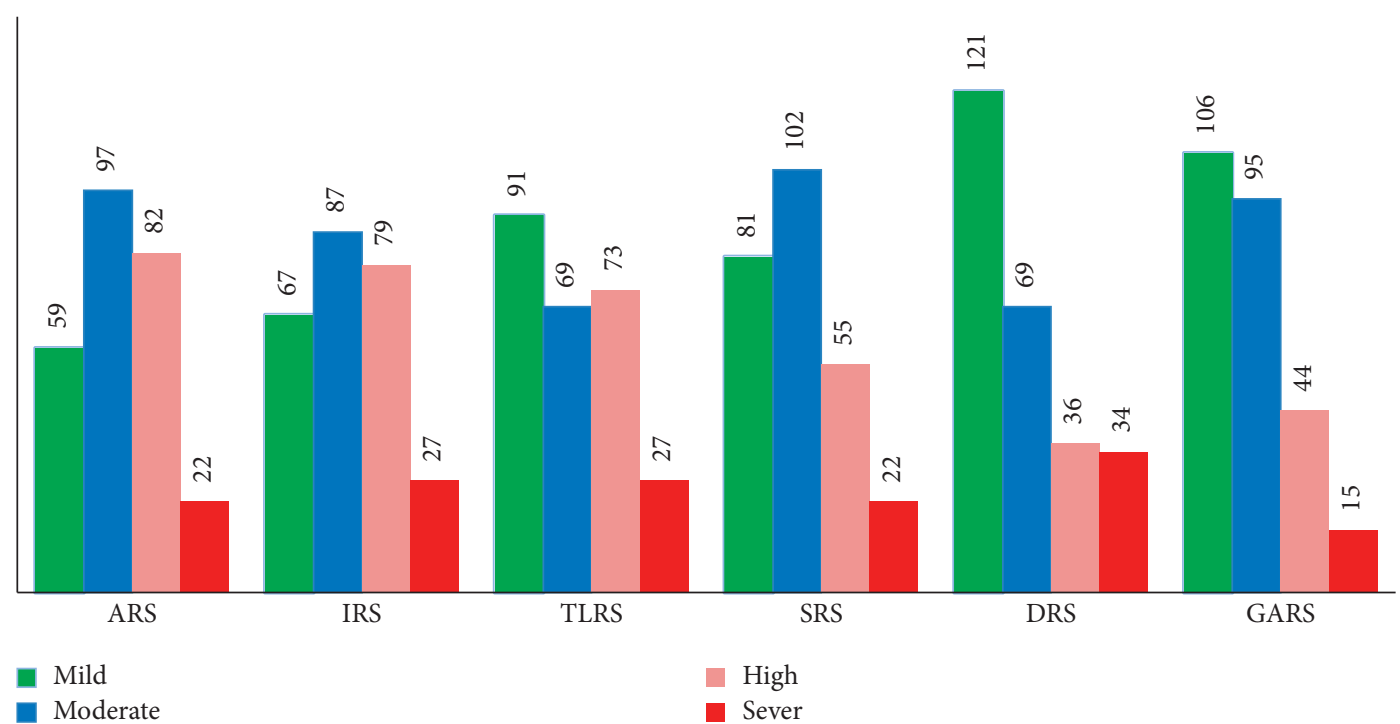

Figure 1: The level of stress caused by six domains of medical student stressors, $N=260$, AU, January 2019.

that is 3.68 times more commonly used in preclinical years than in the clinical years (95\% Cl: 1.18-11.49). Furthermore, in the preclinical year, religion was 3.79 times more commonly used than compared to the clinical years $(95 \% \mathrm{Cl}$ : 1.03-13.91). Additionally, compared to the clinical years, self-blame was 2.64 times more commonly used in the preclinical years (95\% Cl: 1.17-5.99). Emotional support was also 2.52-time more common cause of stress in the preclinical years as compared with clinical years $(95 \% \mathrm{Cl}$ : 1.06-5.99). 
TABLE 3: Rank of coping strategies according to mean score as rated by study participants in relation to gender, $N=260$, AU, January 2019.

\begin{tabular}{|c|c|c|c|}
\hline \multirow{2}{*}{ Scale } & \multicolumn{2}{|c|}{ Sex } & \multirow{2}{*}{ Total $(N=260)$ mean $(S D)$} \\
\hline & Male mean $(\mathrm{SD})$ & Female mean $(\mathrm{SD})$ & \\
\hline Self-distraction & $5.09(1.41)$ & $5.06(1.69)$ & $5.08(1.52)$ \\
\hline Active coping* & $5.56(1.71)$ & $5.46(1.82)$ & $5.52(1.75)$ \\
\hline Denial & $4.12(1.65)$ & $4.07(1.80)$ & $4.10(1.70)$ \\
\hline Substance use* & $3.49(1.75)$ & $3.44(1.92)$ & $3.47(1.81)$ \\
\hline Use of emotional support ${ }^{* *}$ & $4.58(1.59)$ & $4.89(1.67)$ & $4.69(1.63)$ \\
\hline Use of instrumental support ${ }^{* *}$ & $4.92(1.66)$ & $5.33(1.82)$ & $5.07(1.73)$ \\
\hline Behavioral disengagement & $4.33(1.73)$ & $4.37(1.81)$ & $4.34(1.75)$ \\
\hline Venting & $4.76(1.34)$ & $4.76(1.67)$ & $4.76(1.47)$ \\
\hline Positive reframing** & $5.24(1.60)$ & $5.65(1.77)$ & $5.39(1.67)$ \\
\hline Planning & $5.35(1.62)$ & $5.43(1.87)$ & $5.38(1.71)$ \\
\hline Humor** & $4.76(1.78)$ & $4.93(1.89)$ & $4.82(1.82)$ \\
\hline Acceptance** & $4.90(1.63)$ & $5.15(1.78)$ & $4.99(1.69)$ \\
\hline Religion** & $5.85(1.76)$ & $6.09(2.00)$ & $5.94(1.85)$ \\
\hline Self-blame ${ }^{* *}$ & $4.57(1.73)$ & $4.78(1.64)$ & $4.65(1.70)$ \\
\hline
\end{tabular}

${ }^{*}$ Male mean scores are significantly higher than female mean scores. ${ }^{* *}$ Female mean scores are significantly higher than male mean scores.

TABLE 4: Bivariate logistic regression of stressor domain with years of medical education, $N=260$, AU, January 2019.

\begin{tabular}{|c|c|c|c|c|}
\hline \multirow{2}{*}{ Educational year } & \multirow{2}{*}{ Total N (\%) } & \multicolumn{2}{|c|}{ ARS } & \multirow{2}{*}{ COR } \\
\hline & & Disagreed N (\%) & Agreed N (\%) & \\
\hline Preclinical year & $128(100.0)$ & $80(62.5)$ & $48(37.5)$ & $0.81(0.50-1.34)$ \\
\hline \multirow[t]{3}{*}{ Clinical year } & $132(100.0)$ & $76(57.6)$ & $56(42.4)$ & $1.00^{*}$ \\
\hline & \multicolumn{4}{|c|}{ IRS } \\
\hline & & Disagreed N (\%) & Agreed N (\%) & \\
\hline Preclinical year & $128(100.0)$ & $75(58.6)$ & $53(41.4)$ & $1.05(0.64-1.73)$ \\
\hline \multirow[t]{3}{*}{ Clinical year } & $132(100.0)$ & $79(59.8)$ & $53(40.2)$ & $1.00^{*}$ \\
\hline & \multicolumn{4}{|c|}{ TLRS } \\
\hline & & Disagreed N (\%) & Agreed N (\%) & \\
\hline Preclinical year & $128(100.0)$ & $69(53.9)$ & $59(46.1)$ & $1.90(1.14-3.15)$ \\
\hline \multirow[t]{3}{*}{ Clinical year } & $132(100.0)$ & $91(68.9)$ & $41(31.1)$ & $1.00^{*}$ \\
\hline & \multicolumn{4}{|c|}{ SRS } \\
\hline & & Disagreed N (\%) & Agreed N (\%) & \\
\hline Preclinical year & $128(100.0)$ & $91(71.1)$ & $37(28.9)$ & $0.94(0.55-1.59)$ \\
\hline \multirow[t]{3}{*}{ Clinical year } & $132(100.0)$ & $92(69.7)$ & $40(30.3)$ & $1.00^{*}$ \\
\hline & \multicolumn{4}{|c|}{ DRS } \\
\hline & & Disagreed N (\%) & Agreed N (\%) & \\
\hline Preclinical year & $128(100.0)$ & $88(68.8)$ & $40(31.3)$ & $1.55(1.09-2.69)$ \\
\hline \multirow[t]{3}{*}{ Clinical year } & $132(100.0)$ & $102(77.3)$ & $30(22.7)$ & $1.00^{*}$ \\
\hline & \multicolumn{4}{|c|}{ GARS } \\
\hline & & Disagreed N (\%) & Agreed N (\%) & \\
\hline Preclinical year & $128(100.0)$ & $99(77.3)$ & $29(22.7)$ & $1.00(0.56-1.78)$ \\
\hline Clinical year & $132(100.0)$ & $102(77.3)$ & $30(22.7)$ & $1.00^{*}$ \\
\hline
\end{tabular}

${ }^{*}$ Reference category. Disagreed: medical students who stated that specific stimulus is not a cause of stress at specific medical education. Agreed: medical students who stated that specific stimulus is a cause of stress at specific medical education. Preclinical year: medical students at the first, second, and third year of medicine. Clinical year: medical students at the fourth, fifth, and sixth (internship) year of medicine.

\section{Discussion}

The main goal and objective of the medical curriculum is to provide competent and safe doctors to the community. However, the mental health problem among university undergraduate students is an important and developing public health concern [57]. Coping strategies are how a person reacts or responses to a stressor [58-61]. Although coping does not directly reduce stress levels, it moderates the impact of stress, according to Lazarus [62]. Equipping undergraduates with the skills necessary to recognize personal distress (to determine when they need to seek assistance) and to develop strategies to promote their own well-being is fundamental to promoting professionalism [63]. Therefore, the main aim of the present study was to evaluate the most common medical students' stressors and coping strategies 
TABle 5: Bivariate logistic regression of coping strategies adopted by year of medical education, $N=260$, AU, January 2019.

\begin{tabular}{|c|c|c|c|c|}
\hline \multirow{2}{*}{ Educational year } & & \multicolumn{2}{|c|}{ Self-distraction } & \multirow{2}{*}{ COR } \\
\hline & & Rarely used N (\%) & Frequently used N (\%) & \\
\hline Preclinical year & $128(100.0)$ & $5(3.9)$ & $123(96.1)$ & $0.38(0.07-1.99)$ \\
\hline \multirow[t]{3}{*}{ Clinical year } & $132(100.0)$ & $2(1.5)$ & $130(98.5)$ & $1.00^{*}$ \\
\hline & \multicolumn{4}{|c|}{ Active coping } \\
\hline & & Rarely used N (\%) & Frequently used N (\%) & \\
\hline Preclinical year & $128(100.0)$ & $3(2.3)$ & $125(97.7)$ & $2.33(0.59-9.23)$ \\
\hline \multirow[t]{3}{*}{ Clinical year } & $132(100.0)$ & $7(5.3)$ & $125(94.7)$ & $1.00^{*}$ \\
\hline & \multicolumn{4}{|c|}{ Denial } \\
\hline & & Rarely used N (\%) & Frequently used N (\%) & \\
\hline Preclinical year & $128(100.0)$ & $23(18.0)$ & $105(82.0)$ & $1.71(0.95-3.09)$ \\
\hline \multirow[t]{3}{*}{ Clinical year } & $132(100.0)$ & $36(27.3)$ & $96(72.7)$ & $1.00^{*}$ \\
\hline & \multicolumn{4}{|c|}{ Substance use } \\
\hline & & Rarely used N (\%) & Frequently used N (\%) & \\
\hline & $128(100.0)$ & $68(53.1)$ & $60(46.9)$ & $0.81(0.50-1.31)$ \\
\hline \multirow[t]{3}{*}{ Clinical year } & $132(100.0)$ & $63(47.7)$ & $69(52.3)$ & $1.00^{*}$ \\
\hline & \multicolumn{4}{|c|}{ Use of instrumental support } \\
\hline & & Rarely used N (\%) & Frequently used N (\%) & \\
\hline Preclinical year & $128(100.0)$ & $6(4.7)$ & $122(95.3)$ & $3.01(1.15-7.89)$ \\
\hline \multirow[t]{3}{*}{ Clinical year } & $132(100.0)$ & $17(12.9)$ & $115(87.1)$ & $1.00^{*}$ \\
\hline & \multicolumn{4}{|c|}{ Behavioral disengagement } \\
\hline & & Rarely used N (\%) & Frequently used N (\%) & \\
\hline Preclinical year & $128(100.0)$ & $17(13.3)$ & $111(86.7)$ & $2.18(1.14-4.15)$ \\
\hline \multirow[t]{3}{*}{ Clinical year } & $132(100.0)$ & $33(25.0)$ & $99(75.0)$ & $1.00^{*}$ \\
\hline & \multicolumn{4}{|c|}{ Venting } \\
\hline & & Rarely used N (\%) & Frequently used N (\%) & \\
\hline Preclinical year & $128(100.0)$ & $6(4.7)$ & $122(95.3)$ & $2.03(0.74-5.59)$ \\
\hline \multirow[t]{3}{*}{ Clinical year } & $132(100.0)$ & $12(9.1)$ & $120(90.9)$ & $1.00^{*}$ \\
\hline & \multicolumn{4}{|c|}{ Positive reframing } \\
\hline & & Rarely used N (\%) & Frequently used N (\%) & \\
\hline Preclinical year & $128(100.0)$ & $5(3.9)$ & $123(96.1)$ & $1.80(0.59-5.53)$ \\
\hline \multirow[t]{3}{*}{ Clinical year } & $132(100.0)$ & $9(6.8)$ & $123(93.2)$ & $1.00^{*}$ \\
\hline & \multicolumn{4}{|c|}{ Planning } \\
\hline & & Rarely used N (\%) & Frequently used N (\%) & \\
\hline Preclinical year & $128(100.0)$ & $5(3.9)$ & $123(96.1)$ & $2.02(0.67-6.07)$ \\
\hline Clinical year & $132(100.0)$ & $10(7.6)$ & $122(92.4)$ & $1.00^{*}$ \\
\hline & & & & \\
\hline & & Rarely used N (\%) & Frequently used N (\%) & \\
\hline Preclinical year & $128(100.0)$ & $17(13.3)$ & $111(86.7)$ & $0.90(0.43-1.87)$ \\
\hline Clinical year & $132(100.0)$ & $16(12.1)$ & $116(87.9)$ & $1.00^{*}$ \\
\hline & & & nce & \\
\hline & & Rarely used N (\%) & Frequently used N (\%) & \\
\hline Preclinical year & $128(100.0)$ & $4(3.1)$ & $124(96.9)$ & $3.68(1.18-11.49)$ \\
\hline Clinical year & $132(100.0)$ & $14(10.6)$ & $118(89.4)$ & $1.00^{*}$ \\
\hline & & & & \\
\hline & & Rarely used N (\%) & Frequently used N (\%) & \\
\hline Preclinical year & $128(100.0)$ & $3(2.3)$ & $125(97.7)$ & $3.79(1.03-13.91)$ \\
\hline Clinical year & $132(100.0)$ & $11(8.3)$ & $121(91.7)$ & $1.00^{*}$ \\
\hline & & & & \\
\hline & & Rarely used N (\%) & Frequently used N (\%) & \\
\hline Preclinical year & $128(100.0)$ & $9(7.0)$ & $119(93.0)$ & $2.64(1.17-5.99)$ \\
\hline Clinical year & $132(100.0)$ & $22(16.7)$ & $110(83.3)$ & $1.00^{*}$ \\
\hline & & Use of & al support & \\
\hline & & Rarely used N (\%) & Frequently used N (\%) & \\
\hline Preclinical year & $128(100.0)$ & $8(6.3)$ & $120(93.8)$ & $2.52(1.06-5.99)$ \\
\hline Clinical year & $132(100.0)$ & $19(14.4)$ & $113(85.6)$ & $1.00^{*}$ \\
\hline
\end{tabular}

\footnotetext{
${ }^{*}$ Reference category.
} 
among undergraduate students enrolled in the Medical School of Arsi University and their association with educational year levels.

Throughout the world, medical education has been reported as one of the most stressful academic curricula [64], and medical students are more prone to different kinds of academic and nonacademic stressors compared to students from other specialties and other persons [65-68]. In the present study, we observed that academic-related followed by interpersonal- and intrapersonal-related stressors are the major stressors faced by students. The reason might be that the undergraduate medical life predisposes a person to several stressors such as academic demands, preexam preparation, inability to cope, helplessness, increased psychological pressures, too much [69], facing new or sometimes difficult schoolwork, lack of self-esteem, and death or loss of loved one(s) [31, 70]. Lack of leisure time, material to be learned, and frequent academic examinations in a competitive environment predispose the students to stress [71]. These students might also be susceptible to additional stressors such as financial issues [30], adaptation to the new environment, being away from home for the first time, and changes in living arrangements [31]. Our present finding is in line with the study conducted by Patil et al. [72], Iqbal et al. [50], and Mehta et al. [73] that found the top three stressors in descending order of occurrence were academics related, intrapersonal and interpersonal related, and group activity related.

In the present study, lack of time to review what has been learned was the top source of stress. The study conducted by Sreeramareddy et al. [33] strengthens the present finding that the most common sources of stress among medical students were the vastness of the courses and the frequency of examinations. Furthermore, studies have revealed that the stressors affecting medical students' well-being seem to be related to medical training, especially academic matters $[51,74-78]$. They found that the top four stressors were tests and examination, time pressure, too much content to be studied, and getting behind in work [51]. Similar results were also reported by other studies [79-82]. However, our present finding is inconsistent with the study reports of Kholoud [83] and Siraj et al. [84] that showed a high level of stress in medical students can be attributed to the course workload, lack of leisure time, shortage of learning materials, and frequent examinations. In Pakistan, the most common stressors among medical students were high parental expectations, frequency of examinations, vastness of academic curriculum, sleeping difficulties, performance in periodic examinations, and worries of the future [85]. Discrepancies stemming from the methodology and type of questionnaire used, could account for this high prevalence obtained by the aforementioned authors. Other possible reasons for the variability could be due to certain differences in the curriculum, teaching facilities, qualification and experience of the instructors, and level of care given to the students.

Coping strategies refer to specific efforts, both behavioral and psychological, that people employ to master, reduce, tolerate, or minimize stress due to undesired events [58-61]. Effective and appropriate coping strategies may minimize the impact of encountered stressful situations on one's wellbeing $[86,87]$. The strategies that the students identified for coping with stress covered almost all categories reported previously [88]. "Active coping" means taking action or exerting efforts to remove or circumvent the stressor. "Acceptance" means accepting the stressful event. "Planning" consists of thinking about how to confront the stressor. "Positive" reframing means making the best of the situation by learning from it. "Denial" is an attempt to reject the reality of a stressful event. "Behavioral disengagement" means giving up or withdrawing efforts to attain a goal [33].

In the present study, the widely employed original COPE questionnaire containing 14 domains of coping strategies was utilized to assess the most common strategies adopted by respondents [56]. In this study, the top coping strategies frequently used by the respondents were religious coping, active coping, positive reframing, and planning strategies. The main coping strategy adopted by the respondents was religion, which was similar to the findings of Al-Sowygh [89], Gade et al. [87], Ahmad et al. [79], Bormann et al. [90], and Muhamad [91]. The use of spirituality and religious practice, according to Rosmarin et al. [92], in the form of an adaptive manner can be helpful to the individual who has a series of psychological distress. This was also reported by Krauss et al. [93] and Watterson and Giesler [94] who stated that religious people posed higher levels of self-control. Thus they are more able to persist in difficult tasks and life situations. However, our result finding was dissimilar with the study findings reported in Malaysia [56], United Arab Emirates [6], and Jordan [95]. In a study in Pakistan [69], sports, music, hanging out with friends, sleeping, or going into isolation were employed in coping with stress. Students in Nepal [33] adopted active coping strategies (positive reframing, planning, acceptance, and active coping) rather than avoidant strategies (denial, alcohol/drug use, and behavioral disengagement). In a qualitative study of Malaysian students [49], common coping strategies adopted by students were regular exercise, praying, counseling, watching cartoons or comedy, practicing meditation, including yoga and tai chi, and listening to soft music. The possible reasons for the variability could be due to certain differences in the curriculum, teaching facilities, and the level of care given to the students.

In this cross-sectional study, we correlated medical students' stressor domain and coping strategies (as independent variables) with preclinical and clinical educational year levels amongst the undergraduate students enrolled in Arsi University College of Health Sciences. In the present study, preclinical year medical students were more exposed to different stressor domains than clinical students. The different studies conducted by different expertise strengthen the present finding [96-99]. The possible reason could be the amount and complexity of the material to be learned in the second year with progressive assessments of anatomy, physiology, and biochemistry that they have to pass to join the next higher level. Additional supportive evidence is the high level of stress, and stress-related illness can be attributed to course workload, lack of leisure time, shortage of learning materials, and frequent examinations [83, 84]. On 
the other hand, senior students developed skills of how to manage stress and stress-related illness than students in the early years [100].

It has been proven that coping mechanisms are essential for individuals perceiving stress [89]. In terms of utilization of different coping mechanisms, in the present study, a significant correlation with the preclinical and clinical educational year levels was found. In particular, the use of instrumental support, behavioral disengagement, acceptance, religion, self-blame, and use of emotional support were common coping strategies utilized by preclinical than clinical students, which have been reported in studies as very adaptive and hasten the recovery from distress [54, 60, 89, 101]. However, our present result finding was dissimilar to studies conducted in the United Kingdom and Jordanian medical students, who have used alcohol, tobacco, and drugs as common coping strategies $[76,95,99,102,103]$. The possible reasons for the variability could be due to geographical and racial differences.

\section{Conclusion}

To sum up, academic-related stressors are the major stressor in medical students. Furthermore, lack of time to review what has been learned, conflict with teacher(s), uncertainty of what is expected, facing illness or death of the patients, unwillingness to study medicine, and need to do well were the top sources of stress. Among coping strategies, "religious coping, active coping, positive reframing, and planning" were found to be the axioms used by most respondents. Inversely, behavioral disengagement, denial, and substance use were found to be the least used coping strategies. This cross-sectional study also showed that preclinical year medical students were more exposed to different stressor domains than clinical students. In terms of utilization of different coping mechanisms, the use of instrumental support, behavioral disengagement, acceptance, religion, self-blame, and use o emotional support were commonly utilized by preclinical than clinical students. Coping strategy sessions for stress management should be routinely held for medical students. Besides stress reduction interventions, the implementation of a structured orientation program that addresses issues such as expectations for each phase, how students are going to be evaluated, how to cope, and how to get through each phase smoothly were recommended. In addition to awareness creation, establishing a students' counseling center in the campus with qualified staff is also highly recommended. Furthermore, academic counseling in the first three years of the courses and stress reduction interventions were recommended. It was recommended to carry out an in-depth study of stressors and their contributing factors among all semesters and other medical colleges. Family or close friend problem (recent death or accident), distance from family, frequency of money sent, and being first from home to go far were not assessed. We also conducted the present study as a preliminary study. However, in future, by incorporating all possible risk factors, studies have to be conducted at large.

\section{Data Availability}

The dataset used/analyzed during the current study is available from the corresponding authors on reasonable request.

\section{Conflicts of Interest}

The authors declare that there are no conflicts of interest regarding the publication of this paper.

\section{Authors' Contributions}

GB and LM participated in the design of the study, data analyses, and manuscript preparation. All authors had an equal contribution and had read and approved the final manuscript.

\section{Acknowledgments}

The authors are grateful to the College of Health Sciences Research and Community Office of Arsi University, all medical students of Arsi University, data collectors, and supervisors for their support and contribution. Furthermore, they also thank Research Square for presenting an earlier version of the document as "preprint" in the following link: https://www.researchsquare.com/article/rs$152511 / \mathrm{v} 1$.

\section{References}

[1] S. McNamara and S. McNamara, Stress in Young People : What's New and what Can We Do? p. 222, Continuum, New York, NY, USA, 2000.

[2] S. Borjalilu, A. Mohammadi, and R. Mojtahedzadeh, "Sources and severity of perceived stress among Iranian medical students," Iranian Red Crescent Medical Journal, vol. 17, Article ID e17767, 2015.

[3] M. Yusoff, "A multi-center study on validity of medical student stressor questionnaire (MMS)," International Medical Journal, vol. 18, pp. 14-18, 2011.

[4] A. Usman, Z. Ahmed, I. Ahmed, and Z. Akbar, "Work stress experienced by the teaching staff of University of the Punjab, Pakistan: antecedents and consequences," International Journal of Business and Social Science, vol. 2, pp. 202-210, 2011.

[5] H. Selye, "Confusion and controversy in the stress field," Journal of Human Stress, vol. 1, no. 2, pp. 37-44, 1975.

[6] A. O. Carter, M. Elzubeir, Y. M. Abdulrazzaq, A. D. Revel, and A. Townsend, "Health and lifestyle needs assessment of medical students in the United Arab Emirates," Medical Teacher, vol. 25, no. 5, pp. 492-496, 2003.

[7] K. Santosh, K. Kudachi, and S. Goudar, "Perceived stress and academic performance among medical students -a cross sectional study," IJBAP, vol. 1, pp. 123-126, 2012.

[8] A. Oku, O. Oku, E. Owoaje, and B. Ikpeme, "Prevalence of stress, stressors and coping strategies among medical students in a Nigerian medical school," African Journal of Medical and Health Sciences, vol. 14, no. 1, pp. 29-34, 2015.

[9] A. Baum, "Stress, intrusive imagery, and chronic distress," Health Psychology, vol. 9, no. 6, pp. 653-675, 1990. 
[10] J. A. Toews, J. M. Lockyer, D. J. Dobson et al., “Analysis of stress levels among medical students, residents, and graduate students at four Canadian schools of medicine," Academic Medicine, vol. 72, no. 11, pp. 997-1002, 1997.

[11] M. C. Rosal, I. S. Ockene, J. K. Ockene, S. V. Barrett, Y. Ma, and J. R. Hebert, "A longitudinal study of students' depression at one medical school," Academic Medicine, vol. 72, no. 6, pp. 542-546, 1997.

[12] T. Stecker, "Well-being in an academic environment," Medical Education, vol. 38, no. 5, pp. 465-478, 2004.

[13] T. H. Mosley, S. G. Perrin, S. M. Neral, P. M. Dubbert, C. A. Grothues, and B. M. Pinto, "Stress, coping, and wellbeing among third-year medical students," Academic Medicine, vol. 69, no. 9, pp. 765-767, 1994.

[14] L. N. Dyrbye, D. V. Power, F. S. Massie et al., "Factors associated with resilience to and recovery from burnout: a prospective, multi-institutional study of US medical students," Medical Education, vol. 44, no. 10, pp. 1016-1026, 2010.

[15] S. A. Santen, D. B. Holt, J. D. Kemp, and R. R. Hemphill, "Burnout in medical students: examining the prevalence and associated factors," Southern Medical Journal, vol. 103, no. 8, pp. 758-763, 2010.

[16] L. N. Dyrbye, C. P. West, D. Satele et al., "Burnout among U.S. Medical students, residents, and early career physicians relative to the general U.S. Population," Academic Medicine, vol. 89, no. 3, pp. 443-451, 2014.

[17] L. N. Dyrbye, M. R. Thomas, J. L. Huntington et al., "Personal life events and medical student burnout: a multicenter study," Academic Medicine, vol. 81, no. 4, pp. 374-384, 2006.

[18] S. L. Ghodasara, M. A. Davidson, M. S. Reich, C. V. Savoie, and S. M. Rodgers, "Assessing student mental health at the vanderbilt university school of medicine," Academic Medicine, vol. 86, no. 1, pp. 116-121, 2011.

[19] L. N. Dyrbye, M. R. Thomas, and T. D. Shanafelt, "Systematic review of depression, anxiety, and other indicators of psychological distress among U.S. And Canadian medical students," Academic Medicine, vol. 81, no. 4, pp. 354-373, 2006.

[20] L. N. Dyrbye, W. Harper, S. J. Durning et al., "Patterns of distress in US medical students," Medical Teacher, vol. 33, no. 10, pp. 834-839, 2011.

[21] L. N. Dyrbye, M. R. Thomas, A. Eacker et al., "Race, ethnicity, and medical student well-being in the United States," Archives of Internal Medicine, vol. 167, no. 19, pp. 2103-2109, 2007.

[22] C. M. L. R. Brazeau, T. Shanafelt, S. J. Durning et al., "Distress among matriculating medical students relative to the general population," Academic Medicine, vol. 89, no. 11, pp. 1520-1525, 2014.

[23] C. K. Smith, D. F. Peterson, B. F. Degenhardt, and J. C. Johnson, "Depression, anxiety, and perceived hassles among entering medical students," Psychology Health \& Medicine, vol. 12, no. 1, pp. 31-39, 2007.

[24] M. R. Hill, S. Goicochea, and L. J. Merlo, "In their own words: stressors facing medical students in the millennial generation," Medical Education Online, vol. 23, p. 1530558, 2018.

[25] M. T. Compton, J. Carrera, and E. Frank, "Stress and depressive symptoms/dysphoria among US medical students," The Journal of Nervous and Mental Disease, vol. 196, no. 12, pp. 891-897, 2008.

[26] K. I. Mavor, K. G. McNeill, K. Anderson, A. Kerr, E. O’Reilly, and M. J. Platow, "Beyond prevalence to process: the role of self and identity in medical student well-being," Medical Education, vol. 48, no. 4, pp. 351-360, 2014.

[27] K. I. Mavor and K. G. McNeill, "Moving toward a complex systems view in medical student well-being research," Medical Education, vol. 48, no. 10, pp. 941-943, 2014.

[28] B. S. Linn and R. Zeppa, "Stress in junior medical students," Academic Medicine, vol. 59, no. 1, pp. 7-12, 1984.

[29] E. Chang, F. Eddins-Folensbee, and J. Coverdale, "Survey of the prevalence of burnout, stress, depression, and the use of supports by medical students at one school," Academic Psychiatry, vol. 36, no. 3, pp. 177-182, 2012.

[30] F. Rab, R. Mamdou, and S. Nasir, "Rates of depression and anxiety among female medical students in Pakistan," Eastern Mediterranean health journal $=$ La revue de sante de la Mediterranee orientale $=$ al-Majallah al-sihhiyah li-sharq almutawassit, vol. 14, pp. 126-133, 2008.

[31] P. B. Kallingappa and S. Asokan, "Psychosocial perspective of first year medical students entered in a professional course-A cross sectional study from davangere, Karnataka," Medical Science, vol. 2, no. 2, pp. 110-116, 2014.

[32] R. K. Agrawal and S. S. Chahar, "Examining role stress among technical students in India," Social Psychology of Education, vol. 10, no. 1, pp. 77-91, 2007.

[33] C. T. Sreeramareddy, P. R. Shankar, V. Binu, C. Mukhopadhyay, B. Ray, and R. G. Menezes, "Psychological morbidity, sources of stress and coping strategies among undergraduate medical students of Nepal," BMC Medical Education, vol. 7, no. 1, p. 26, 2007.

[34] S. Deb, E. Strodl, and J. Sun, "Academic stress, parental pressure, anxiety and mental health among Indian high school students," International Journal of Psychology and Behavioral Sciences, vol. 5, pp. 26-34, 2015.

[35] R. P. Ang and V. S. Huan, "Relationship between academic stress and suicidal ideation: testing for depression as a mediator using multiple regression," Child Psychiatry and Human Development, vol. 37, no. 2, pp. 133-143, 2006.

[36] M. María, L. Sandra, L. Cristina, L. Irene, M. Lidia, and S. Ángela, "Stress perception in Nursing students facing their clinical practices,” Enfermería Global, vol. 31, pp. 244-253, 2013.

[37] L. N. Dyrbye, M. R. Thomas, and T. D. Shanafelt, "Medical student distress: causes, consequences, and proposed solutions," Mayo Clinic Proceedings, vol. 80, no. 12, pp. 1613-1622, 2005.

[38] S. Folkman, "Personal control and stress and coping processes: a theoretical analysis," Journal of Personality and Social Psychology, vol. 46, no. 4, pp. 839-852, 1984.

[39] U. Rout and J. Rout, Stress and General Practitioners, Kluwer Academic Publishers, London, UK, 1993.

[40] R. Walton, A Comparison of Perceived Stress Levels and Coping Styles of Junior and Senior Students in Nursing and Social Work Programs [Internet], West Virginia Marshall University, Huntington, WV, USA, 2002.

[41] J. Lee and A. V. Graham, "Students' perception of medical school stress and their evaluation of a wellness elective," Medical Education, vol. 35, no. 7, pp. 652-659, 2001.

[42] K. S. Franco, M. B. Tamburrino, B. T. Carroll, A. Somani, and S. M. Wagner, "Reducing the stress of a medical education: two approaches," Ohio Medicine : Journal of the Ohio State Medical Association, vol. 83, pp. 691-692, 1987.

[43] B. Bewick, G. Koutsopoulou, J. Miles, E. Slaa, and M. Barkham, "Changes in undergraduate students' psychological well-being as they progress through university," Studies in Higher Education, vol. 35, no. 6, pp. 633-645, 2010. 
[44] M. Stern, S. Norman, and C. Komm, "Medical students' differential use of coping strategies as a function of stressor type, year of training, and gender," Behavioral Medicine, vol. 18, no. 4, pp. 173-180, 1993.

[45] A. N. Supe, "A study of stress in medical students at Seth G.S. Medical College," Journal of Postgraduate Medicine, vol. 44, pp. 1-6, 1998.

[46] T. M. Wolf, "Stress, coping and health: enhancing well-being during medical school," Medical Education, vol. 28, no. 1, pp. 8-17, 1994.

[47] N. Seyedfatemi, M. Tafreshi, and H. Hagani, "Experienced stressors and coping strategies among Iranian nursing students," BMC Nursing, vol. 6, no. 1, p. 11, 2007.

[48] Arsi University, Continuous Professional Development, Arsi University, Rustavi, Georgia, 2020, https://www.arsiun.edu.et/ index.php/2015-11-05-17-37-13/2015-11-05-18-48-17/collegeof-health-sciences.

[49] A. Redhwan, A. Sami, A. Karim, R. Chan, and M. Zaleha, "Stress and coping strategies among Management and Science University students: a qualitative study," International Medical Journal, vol. 8, pp. 11-15, 2009.

[50] S. Iqbal, S. Gupta, and E. Venkatarao, "Stress, anxiety and depression among medical undergraduate students and their socio-demographic correlates," Indian Journal of Medical Research, vol. 141, pp. 354-357, 2015.

[51] S. Muhamad, F. Ahmad, and M. Yaacob, "The development and validity of the medical student stressor questionnaire (MSSQ)," ASEAN Journal of Psychiatry, vol. 11, pp. 1-12, 2010.

[52] S. M. Downing, "Reliability: on the reproducibility of assessment data," Medical Education, vol. 38, no. 9, pp. 1006-1012, 2004.

[53] S. Muhamad, "A confrmatory factor analysis study on the Medical Student Stressor Questionnaire among Malaysian medical students," Education in Medicine Journal, vol. 3, pp. e44-53, 2011.

[54] C. S. Carver, "You want to measure coping but your protocol' too long: consider the brief cope," International Journal of Behavioral Medicine, vol. 4, no. 1, pp. 92-100, 1997.

[55] I. Olssøn, A. Mykletun, and A. Dahl, "The Hospital Anxiety and Depression Rating Scale: a cross-sectional study of psychometrics and case fnding abilities in general practice," BMC Psychiatry, vol. 14, p. 46, 2005.

[56] S. Al-Dubai, R. Al-Naggar, A. Mustafa, and G. Krishna, "Stress and coping strategies of students in a medical faculty in Malaysia," Malaysian Journal of Medical Sciences, vol. 18, pp. 57-64, 2011.

[57] A. Syed, S. S. Ali, and M. Khan, "Frequency of depression, anxiety and stress among the undergraduate physiotherapy students," Pakistan journal of medical sciences, vol. 34, pp. 468-471, 2018.

[58] D. Rosenhan and M. Seligman, Abnormal Psychology,pp. 463-464, Norton, 2nd edition, New York, NY, USA, 1989.

[59] H. Selye, Stress without Distress, pp. 12-13, Harper \& Row, New York, NY, USA, 1974.

[60] D. Myers, "Stress and health," in Exploring Psychology,Worth Publishers, 6th edition, New York, NY, USA, 2005.

[61] R. S. Lazarus, "Theory-based stress measurement," Psychological Inquiry, vol. 1, no. 1, pp. 3-13, 1990.

[62] R. S. Lazarus, "Coping theory and research: past, present, and future," Psychosomatic Medicine, vol. 55, no. 3, pp. 234-247, 1993.
[63] M. Dahlin, N. Joneborg, and B. Runeson, "Stress and depression among medical students: a cross-sectional study," Medical Education, vol. 39, no. 6, pp. 594-604, 2005.

[64] A. Saeed, A. Bahnassy, N. Al-Hamdan, F. Almudhaibery, and A. Alyahya, "Perceived stress and associated factors among medical students," Journal of Family and Community Medicine, vol. 23, no. 3, pp. 166-171, 2016.

[65] V. Padmanathan, H. Deepashini, and J. Ali, "Education stress and coping strategies among physiotherapy students," International Journal of Health Sciences, vol. 3, pp. 7-16, 2013.

[66] M. Sani, M. Mahfouz, I. Bani, A. Alsomily, D. Alagi, and N. Alsomily, "Prevalence of stress among medical students in jizan university, kingdom of Saudi arabia," Gulf Medical Journal, vol. 1, pp. 19-25, 2012.

[67] J. P. Kohn and G. H. Frazer, "An academic stress scale: identification and rated importance of academic stressors," Psychological Reports, vol. 59, no. 2, pp. 415-426, 1986.

[68] R. Misra and M. McKean, "College students' academic stress and its relation to their anxiety, time management, and leisure satisfaction," American Journal of Health Studies, vol. 16, pp. 41-51, 2000.

[69] B. Shaikh, A. Kahloon, M. Kazmi et al., "Students, stress and coping strategies: a case of Pakistani medical school," Education for Health: Change in Learning \& Practice, vol. 17, no. 3, pp. 346-353, 2004.

[70] H. Liaqat, U. Choudry, A. Altaf, J. Sauleh, S. Rahman, A. Choudry et al., "Deranged mental homeostasis in medical students: evaluation of depression anxiety and stress among home and hostel students," Acta Psychopathology, vol. 3, p. 1, 2017.

[71] N. Sohail, "Stress and academic performance among medical students," Journal of the College of Physicians and Surgeons-Pakistan : JCPSP, vol. 23, pp. 67-71, 2013.

[72] S. P. Patil, S. Sadhanala, M. U. Srivastav, and S. S. BansodeGokhe, "Study of stressors among undergraduate medical students of a teaching medical institution," International Journal of Community Medicine And Public Health, vol. 4, no. 9, pp. 3151-3154, 2017.

[73] P. Mehta, K. Thekdi, M. Rokad, K. Patel, A. Bhadu, and P. Sorani, "Exploratory study to access anxiety, depression and stress among medical students, freshly starting their medical education in a medical College. Scholars," Journal of Applied Medical Sciences, vol. 1, pp. 819-822, 2013.

[74] R. Saipanish, "Stress among medical students in a Thai medical school," Medical Teacher, vol. 25, no. 5, pp. 502-506, 2003.

[75] M. Aktekin, T. Karaman, Y. Y. Senol, S. Erdem, H. Erengin, and M. Akaydin, "Anxiety, depression and stressful life events among medical students: a prospective study in Antalya, Turkey," Medical Education, vol. 35, no. 1, pp. 12-17, 2001.

[76] E. A. Guthrie, D. Black, C. M. Shaw, J. Hamilton, F. H. Creed, and B. Tomenson, "Embarking upon a medical career: psychological morbidity in first year medical students," Medical Education, vol. 29, no. 5, pp. 337-341, 1995.

[77] D. M. Kaufman, V. Day, and D. Mensink, "Stressors in lstyear medical school: comparison of a conventional and problem-based curriculum," Teaching and Learning in Medicine, vol. 8, no. 4, pp. 188-194, 1996.

[78] D. Kaufman, V. Day, and D. Mensink, "Stressors in Medical School: relation to curriculum format and year of study," Teaching and Learning in Medicine, vol. 10, pp. 188-194, 1998.

[79] M. S. Ahmad, M. M. Md Yusoff, and I. Abdul Razak, "Stress and its relief among undergraduate dental students in 
Malaysia," Southeast Asian Journal of Tropical Medicine and Public Health, vol. 42, pp. 996-1004, 2011.

[80] H. W. Elani, P. J. Allison, R. A. Kumar, L. Mancini, A. Lambrou, and C. Bedos, "A systematic review of stress in dental students," Journal of Dental Education, vol. 78, no. 2, pp. 226-242, 2014.

[81] G. Muneer, S. Syed, J. Yong, S. Ahmed, P. Wong, and S. Ahmad, "Perceived sources of stress among Malaysian dental students," International Journal of Medical Education, vol. 6, pp. 56-61, 2015.

[82] L. A. Telang, J. T. Nerali, A. Telang, and P. V. K. Chakravarthy, "Perceived sources of stress among Malaysian dental students," European Journal of General Dentistry, vol. 2, no. 03, pp. 300-307, 2013.

[83] A. Kholoud, "Prevalence of stressors among female medical students Taibah University, Saudi Arabia," Journal of Taibah University Medical Sciences, vol. 5, pp. 110-119, 2010.

[84] H. Siraj, A. Salam, R. Roslan, N. Hasan, T. Jin, and M. Othman, "Stress and its association with the academic performance of undergraduate fourth year medical students at Universiti Kebangsaan Malaysia," The International Medical Journal Malaysia, vol. 13, pp. 19-24, 2014.

[85] M. Shah, S. Hasan, S. Malik, and C. T. Sreeramareddy, "Perceived stress, sources and severity of stress among medical undergraduates in a Pakistani Medical school," BMC Medical Education, vol. 10, no. 1, p. 2, 2010.

[86] C. L. Park and N. E. Adler, "Coping style as a predictor of health and well-being across the first year of medical school," Health Psychology, vol. 22, no. 6, pp. 627-631, 2003.

[87] S. Gade, S. Chari, and M. Gupta, "Perceived stress among medical students: to identify its sources and coping strategies," Archives of Medicine and Health Sciences, vol. 2, no. 1, pp. 80-86, 2014.

[88] S. K. Redwood and M. H. Pollak, "Student-led stress management program for first-year medical students," Teaching and Learning in Medicine, vol. 19, no. 1, pp. 42-46, 2007.

[89] Z. H. Al-Sowygh, "Academic distress, perceived stress and coping strategies among dental students in Saudi Arabia," The Saudi Dental Journal, vol. 25, no. 3, pp. 97-105, 2013.

[90] J. E. Bormann, T. L. Smith, S. Becker et al., "Efficacy of frequent mantram repetition on stress, quality of life, and spiritual well-being in veterans: a pilot study," Journal of Holistic Nursing : Official Journal of the American Holistic Nurses' Association, vol. 23, pp. 395-414, 2005.

[91] S. Muhamad, "Stress, stressors and coping strategies among secondary school students in a Malaysian Government Secondary School: initial Findings," ASEAN Journal of Psychiatry, vol. 11, pp. 1-15, 2010.

[92] D. H. Rosmarin, K. I. Pargament, S. Pirutinsky, and A. Mahoney, "A randomized controlled evaluation of a spiritually integrated treatment for subclinical anxiety in the Jewish community, delivered via the Internet," Journal of Anxiety Disorders, vol. 24, no. 7, pp. 799-808, 2010.

[93] S. Krauss, Development of the Muslim Religiosity-Personality Inventory for Measuring the Religiousity of Malaysia Muslim Youth, [Unpublished Ph.D. Thesis]. [Serdang], Universiti Putra Malaysia, Seri Kembangan, Malaysia, 2005.

[94] K. Watterson and R. B. Giesler, "Religiosity and self-control: when the going gets tough, the religious get self-regulating," Psychology of Religion and Spirituality, vol. 4, no. 3, pp. 193-205, 2012.

[95] Z. M. Bataineh, T. A. Hijazi, and M. F. A. Hijleh, "Attitudes and reactions of Jordanian medical students to the dissecting room," Surgical and Radiologic Anatomy, vol. 28, no. 4, pp. 416-421, 2006.

[96] L. Melaku, A. Mossie, and A. Negash, "Stress among medical students and its association with substance use and academic performance," Journal of Biomedical Education, vol. 2015, Article ID 149509, 9 pages, 2015.

[97] S. N. Inam, A. Saqib, and E. Alam, "Prevalence of anxiety and depression among medical students of private university," JPMA. The Journal of the Pakistan Medical Association, vol. 53, pp. 44-47, 2003.

[98] M. S. Khan, S. Mahmood, A. Badshah, S. U. Ali, and Y. Jamal, "Prevalence of depression, anxiety and their associated factors among medical students in Karachi, Pakistan," JPMA. The Journal of the Pakistan Medical Association, vol. 56, pp. 583-586, 2006.

[99] E. A. Guthrie, D. Black, C. M. Shaw, J. Hamilton, F. H. Creed, and B. Tomenson, "Psychological stress in medical students: a comparison of two very different university courses," Stress Medicine, vol. 13, no. 3, pp. 179-184, 1997.

[100] T. Alvi, F. Assad, M. Ramzan, and F. A. Khan, "Depression, anxiety and their associated factors among medical students," Journal of the College of Physicians and Surgeons-Pakistan : JCPSP, vol. 20, pp. 122-126, 2010.

[101] C. S. Carver, M. F. Scheier, and J. K. Weintraub, "Assessing coping strategies: a theoretically based approach," Journal of Personality and Social Psychology, vol. 56, pp. 267-283, 1989.

[102] J. Firth, "Levels and sources of stress in medical students," $B M J$, vol. 292, no. 6529, pp. 1177-1180, 1986.

[103] C. H. Ashton and F. Kamali, "Personality, lifestyles, alcohol and drug consumption in a sample of British medical students," Medical Education, vol. 29, no. 3, pp. 187-192, 1995. 